\title{
CrimRxiv
}

\section{Explaining offenders'}

longitudinal product-

specific target selection

through changes in

disposability, availability,

and value: an open-source

intelligence web-scraping

approach

Liam Quinn, Joseph Clare, Jade Lindley, Frank Morgan

Published on: Feb 24, 2022

DOI: 10.21428/cb6ab371.5f163911

License: Creative Commons Attribution 4.0 International License (CC-BY 4.0). 
Explaining offenders' longitudinal product-specific target selection through changes in disposability, availability, and value: an open-source intelligence web-scraping approach 